\title{
Vortex search algorithm for designing hybrid active power filter
}

\author{
Chau Minh Thuyen ${ }^{1}$, Truong Khac Tung ${ }^{2}$, Nguyen Hoai Phong ${ }^{3}$ \\ ${ }^{1,3}$ Faculty of Electrical Engineering Technology, Industrial University of Ho Chi Minh City, Vietnam \\ ${ }^{2}$ Division of Computational Mathematics and Engineering, Institute for Computational Science, \\ Ton Duc Thang University, Vietnam \\ ${ }^{2}$ Faculty of Civil Engineering, Ton Duc Thang University, Vietnam
}

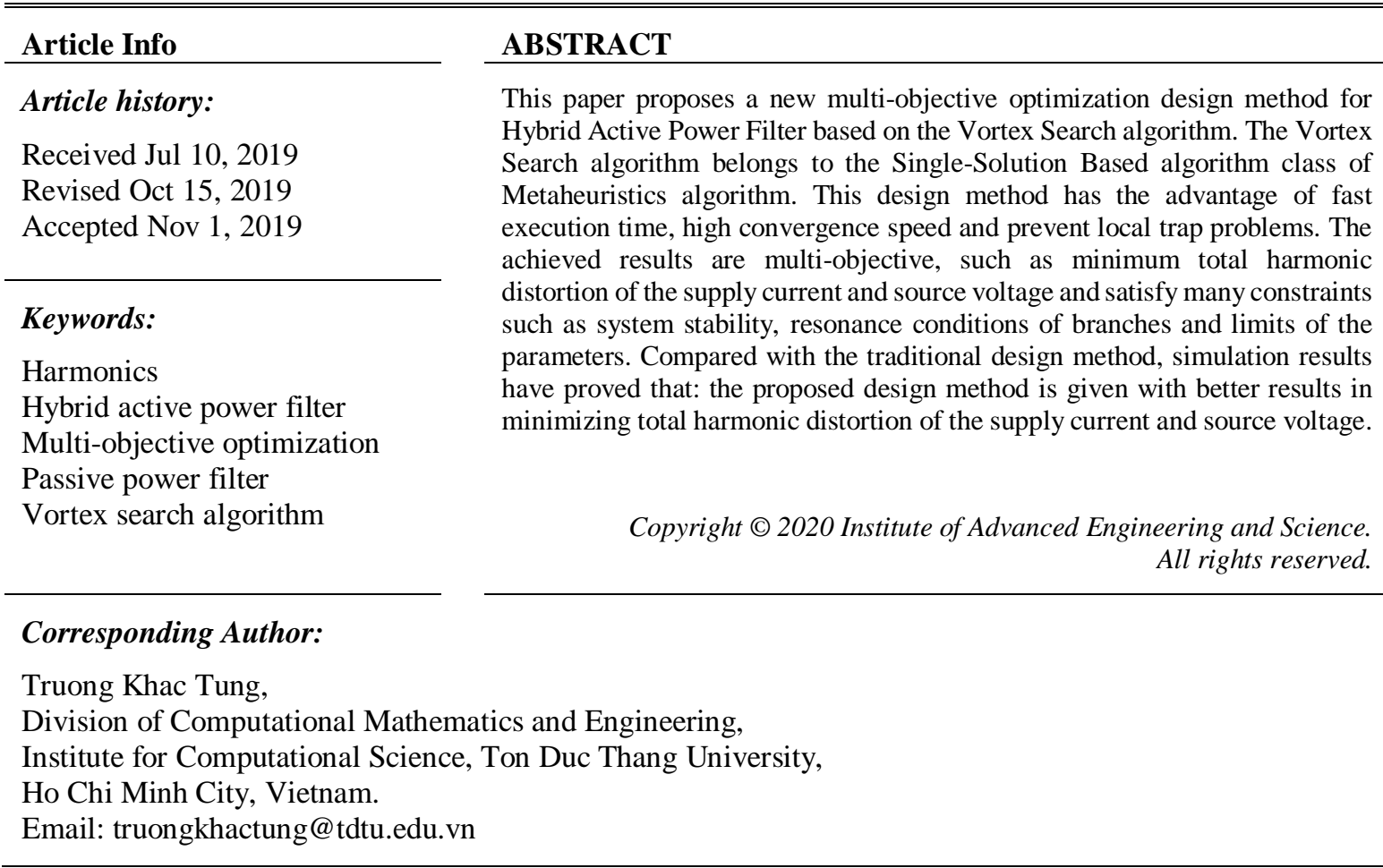

\section{INTRODUCTION}

In power systems, the hybrid active power filter (HAPF) is considered as the most effective solution in harmonic filtering and reactive power compensation [1-3]. However, the working efficiency of HAPF depends on many factors such as: correct determination of the parameters [4], control method [5-8], harmonic current detection method $[9,10]$, control strategy $[11,12]$ and stabilization of the DC-bus for the inverter [13, 14]. In which, the correct calculation of the parameters of the HAPF is the most important factor, it decided to work efficiency of HAPF.

The structure of a HAPF includes: power circuit part and control circuit part. Therefore, the parameters that need to be designed for HAPF are the parameters of the above circuit parts. Until now, the multi-objective optimization studies of HAPF can be summarized as follows: The application of the genetic algorithm to multi-objective optimization design for passive power filter (PPF) is proposed by [15]. Another algorithm is also commonly used for HAPF design, called the Particle Swarm Optimization (PSO) algorithm. The PSO algorithm is used to design an APF in a four-wire three-phase system in case of balanced and unbalanced loads but only consider to design for PPFs and optimization for the APF but not for the HAPF [1619]. Another study to multi-objective design on HAPF is using an ant colony and bat algorithm [20, 21] but only aslo multi-objective optimization design for PPFs. Ahmed Faheem Zobaa has used the Fortran Feasible Sequential Quadratic Programming algorithm to solve the multi-objective optimization problem for HAPF with the aim of finding PPF parameters [22].

In summary, the previous research on multi-objective optimization for HAPF was the only multiobjective optimization design of power circuit parts (parameters of the PPF). Meanwhile, the optimized design 
for the parameters of the control circuit part has not been studied and the calculation of the above parameters without considering the condition of the system stability.

To overcome this disadvantage, in this paper, the Routh's stability standard is used to check the stable condition of the HAPF system. The Vortex search is a new optimization algorithm, that is effecty in solving global optimization [23, 24]. Then a new multi-objective optimization design, based on the Vortex Search (VS) algorithm is proposed to determine the best set of parameters for the HAPF. The proposed algorithm has the advantage of finding a quick solution with little loops. The results achieved will be global optimization such as the minimum of the total harmonic distortion (THD) of the supply current and source voltage and satisfy the stable conditions.

The structure of the paper is divided into five parts. Part 1 is an overview introduction of the issues that need to be investigated. Part 2 is a new multi-objective optimization design method for Hybrid Active Power Filter. The simulation results and discussion are presented in Part 3, and the conclusions are summarized in Part 4.

\section{NEW MULTI-OBJECTIVE OPTIMIZATION DESIGN METHOD FOR HYBRID ACTIVE POWER FILTER}

The single-phase equivalent circuit of the selected Hybrid Active Power Filter topology is shown in Figure 1. It consists of five parts: $U_{s}$ and $Z_{s}$ are the source voltage and the resistance of the source. $C_{1}$ and $L_{1}$ are capacitance and inductance at the fundamental frequency. $C_{F}$ is the added capacitance to compensate for reactive power. $L_{0}$ is the output filter of the voltage source inverter.

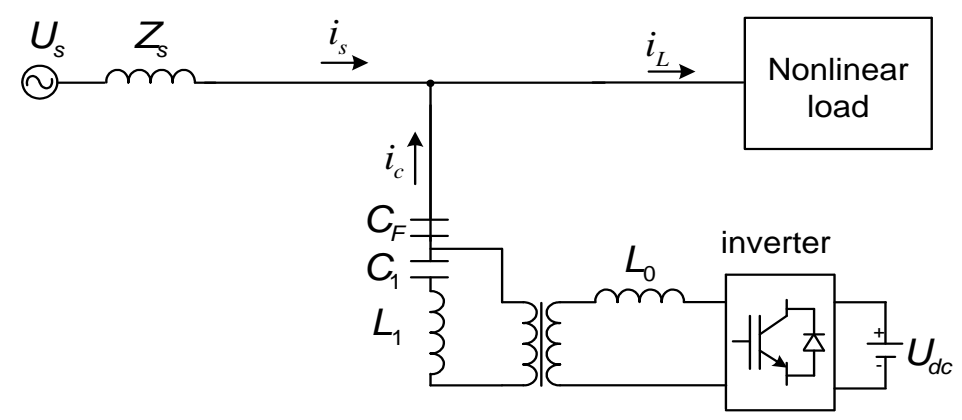

Figure 1. Single-phase equivalent circuit of the selected hybrid active power filter

Starting from the load current $i_{L}\left(i_{L a}, i_{L b}, i_{L c}\right)$, we use p-q instantaneous power theory [9], [10] to separate the harmonic component of the load current as $i_{L h a}, i_{L h b}, i_{L h c}$. These components will be chosen as the reference components and they are compared to the compensate current components into the grid $i_{c}$ of HAPF are $i_{c a}, i_{c b}$, and $i_{c c}$. The error of the above comparison will be taken through the PI (Proportional - Integral) controller, through pulse width modulation to control ON-OFF switches of the inverter to generate compensate signals on the grid. Thus, the inverter will be controlled to produce a signal similar to the harmonic current signal generated from the load.

The parameters of both the power circuit part and the control circuit part need to be determined in the model in Figure 1 including passive circuit $C_{F}-C_{l}-L_{l}$ in which branch $C_{l}-L_{l}$ resonates at the fundamental frequency, output filter $L_{0}$, voltage bus-DC of inverter $U_{d c}$, parameters of PI controller are $K_{p}$ and $K_{i}$. Currently, these parameters are usually determined based on experience and local calculations, regardless of the stability of the system. Therefore, this paper proposes a new multi-objective optimization design method that has considered the stability of the system.

\subsection{Constraints and Objective Function}

System stability constraint: Based on the transfer function of the HAPF system in [1], we have the characteristic equation of the control transfer function as follows:

$$
D(s)=a_{6} s^{6}+a_{5} s^{5}+a_{4} s^{4}+a_{3} s^{3}+a_{2} s^{2}+a_{1} s^{1}
$$

According to Routh's stability standard, the following conditions must be satisfied. 


$$
\left\{\begin{array}{r}
a_{5} a_{4}-a_{6} a_{3}>0 \\
a_{3} a_{2}-a_{4} a_{1}>0 \\
b_{1} a_{3}-a_{5} b_{3}>0 \\
b_{2} b_{3}-b_{1} b_{4}>0 \\
a_{1} b_{3} \quad>0
\end{array}\right.
$$

With: $b_{1}=\frac{a_{5} a_{4}-a_{6} a_{3}}{a_{5}} ; b_{3}=\frac{a_{3} a_{2}-a_{4} a_{1}}{a_{3}} ; b_{2}=\frac{b_{1} a_{3}-a_{5} b_{3}}{b_{1}} ; b_{4}=\frac{b_{3} a_{1}}{b_{3}} ; c_{1}=\frac{b_{3} b_{2}-b_{1} b_{4}}{b_{2}} ; c_{2}=b_{4}$;

$a_{6}=T_{i} T L_{1} C_{1} C_{F}\left(L_{1} L_{s}+L_{1} L_{0}+L_{1} L_{0} L_{s}\right) ; a_{5}=T_{i} T\left(R_{s} L_{1} C_{1} C_{F}+R_{s} L_{0} C_{1} C_{F}\right)+T_{i}\left(C_{1} C_{F} L_{1} L_{s}+L_{1} L_{0} C_{1} C_{F}+C_{1} C_{F} L_{0} L_{s}\right) ;$

$\left.a_{4}=T_{i} T\left(L_{0} C_{F}+L_{s} C_{F}+L_{1} C_{1}+L_{0} C_{1}\right)+T_{i}\left(R_{s} L_{1} C_{1} C_{F}+R_{s} L_{0} C_{1} C_{F}\right)+C_{F} C_{1} L_{1} K_{i n v} K T_{i}\right)$;

$a_{3}=T_{i} T C_{F} R_{s}+T_{i}\left(L_{0} C_{F}+L_{s} C_{F}+L_{1} C_{1}+L_{0} C_{1}\right)+K K_{i n v} C_{1} L_{1} C_{F} ; a_{2}=T_{i} T+T_{i} C_{F} R_{s}+T_{i} K K_{i n v} C_{F} ; a_{1}=T_{i}+K K_{i n v} C_{F}$;

$T=0.01 \mathrm{~ms} ; T_{i}=0.00001 ; K=10 ; K_{i n v}=1$

Constraints on resonance conditions in PPFs: The $L$ and $C$ parameters in a branch must be resonated at a certain frequency.

$$
\omega_{n} L=\frac{1}{\omega_{n} C}
$$

Constraint of passive power filter values: passive power filter values must be positive and meet system stability and resonance conditions.

$$
0<\left(R_{i}, L_{i}, C_{i}\right) \leq\left(R_{\max }, L_{\max }, C_{\max }\right)
$$

the values of $R_{\max }, L_{\max }$, and $C_{\max }$ are determined under stable conditions (2).

PPFs must be compensated with a maximum capacity but not exceed the required maximum limit

$$
Q_{c \min } \leq Q_{c} \leq Q_{c \max }
$$

constraint of DC-bus voltage value:

$$
0<U_{d c}<U_{d c-\max }
$$
conditions (2).

Controller parameters constraints: Controller parameters must be positive and satisfy system stability

$$
0<K_{p}<K_{\text {pmax }} ; 0<K_{i}<K_{\text {imax }}
$$

according to [5], the value of $K_{p}$ and $K_{i}$ is too small or too large to cause system instability.

Objective function: we consider the main objective as follows:

$$
F=\min \left(T H D i_{s}+T H D u_{s}\right)
$$

\subsection{Vortex Search Algorithm and Its Application for Designing Hybrid Active Power Filter}

The Vortex search algorithm is first proposed in [23, 24]. Let us consider a two-dimensional optimization problem. In a two-dimensional case, a vortex pattern can be modelled by a number of nested circles. Here, the largest circle of the vortex is first centered on the search space, where the initial center can be calculated using (9). A description of the VS algorithm is also provided in algorithm 1.

$$
\begin{aligned}
& \mu_{0}=\frac{\text { upperlimit }+ \text { lowerlimit }}{2} \\
& r_{0} \approx \sigma_{0}=\frac{\max (\text { upperlimit })-\min (\text { lowerlimit })}{2}
\end{aligned}
$$


The solutions that exceed the boundaries are shifted into the boundaries, as in (11).

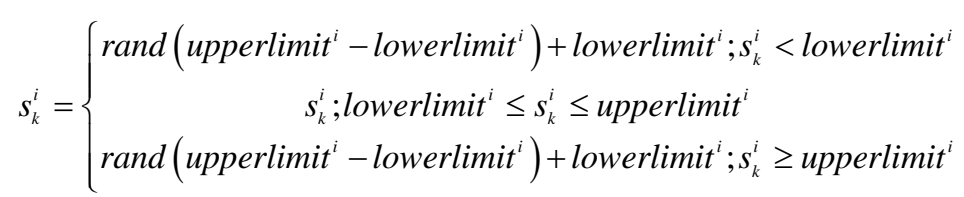

The initial radius $r_{0}$ can be calculated with (10). Because $a_{0}=1$, the resulting function value is $1(x)$ gammaincinv $\left(x, a_{0}\right) \approx 1$, which means $r_{0} \approx \sigma_{0}$ as indicated before.

$$
r_{0}=\sigma_{0} \cdot\left(\frac{1}{x}\right) \cdot \operatorname{gammaincinv}\left(x, a_{0}\right)
$$

by methods of (12), the initial radius value $r_{0}$ can be computed as $r_{0} \approx 10$. Radius at each iteration is calculated by using (13).

$$
r_{t}=\sigma_{0} \cdot\left(\frac{1}{x}\right) \cdot \operatorname{gammaincinv}\left(x, a_{t}\right)
$$

In this algorithm paramater $a_{t}$ of the inverse incomplete gamma function defines the resolution of the search. By equally sampling values within $[1,0]$ interval at a certain step size, the resolution of the search can be adjusted. For this purpose, at generation $\mathrm{t}, a_{\mathrm{t}}$ is obtained by using (14).

$$
a_{t}=a_{0}-\frac{t}{\text { MaxInt }}
$$

where $a_{0}$ is selected as $a_{0}=1$ to ensure full coverage of the search space at the first iteration, $t$ is the iteration index, and MaxItr represents the maximum number of iterations.

Vortex Search algorithm flowchart for designing HAPF is shown as in Figure 2.

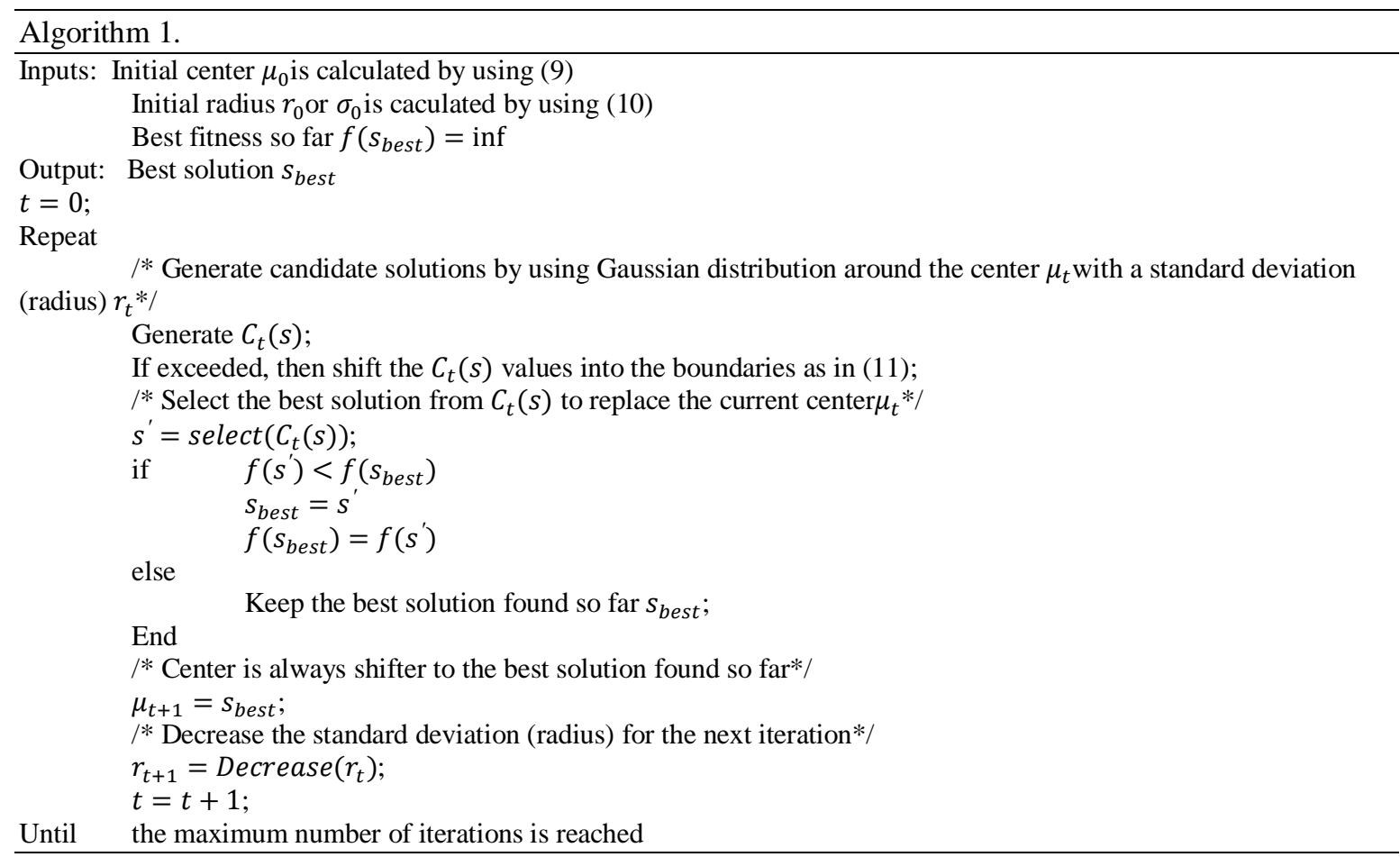




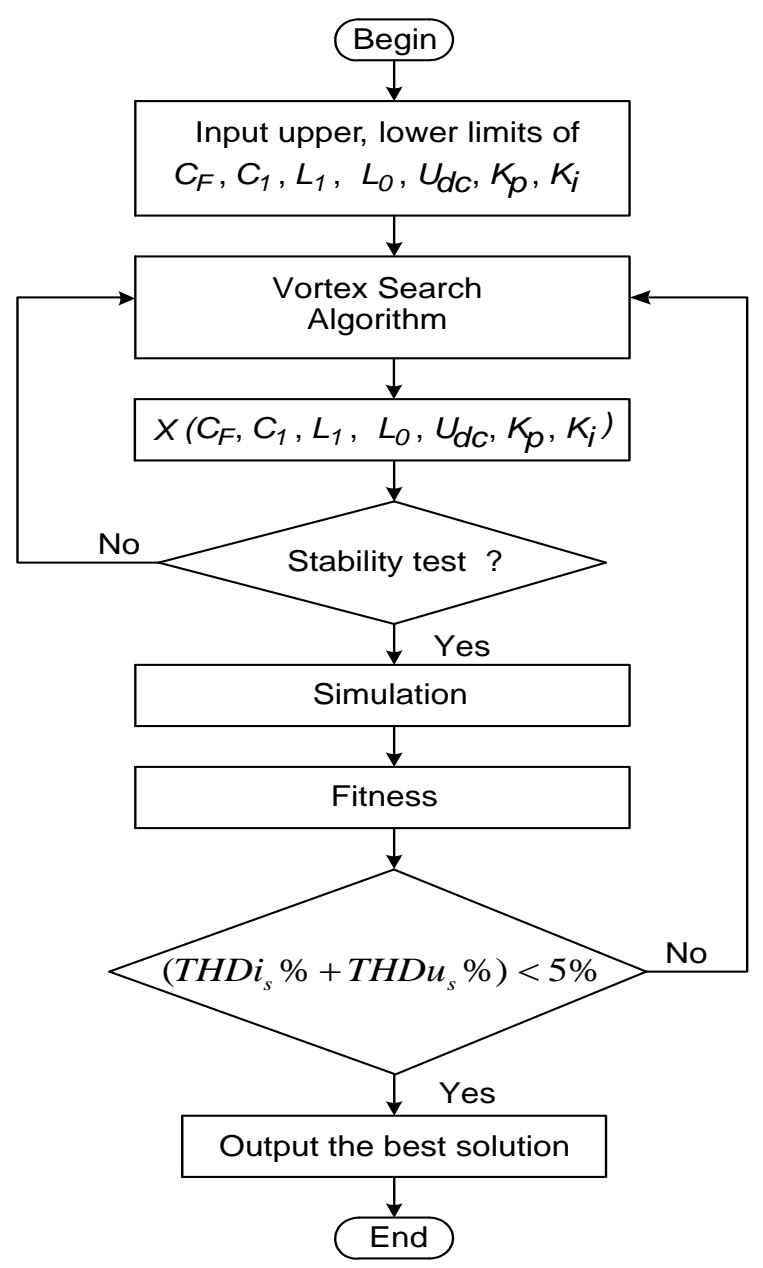

Figure 2. Vortex search algorithm flowchart for designing HAPF

Application of the VS Algorithm for Hybrid Active Power Filter Design is described as follows: The algorithm begins by entering the upper and lower limit values of the parameters to be searched based on the constraints in the formulas from (3) to (7), then these limits will be included in the VS algorithm to find a set of parameters to be searched $X\left(C_{F}, C_{1}, L_{1}, L_{0}, U_{d c}, K_{p}, K_{i}\right)$. Next, these parameters will be checked for stable conditions using formula (2), if not stable condition, return to VS algorithm to create a new set of parameters, if stable condition is satisfied, these parameters will be setup into the HAPF simulation model to find the $T H D i_{s} \%$ and $T H D u_{s} \%$ values.

The value of $T H D i_{s} \%$ must be less than $\varepsilon_{1}$ and $T H D u_{s} \%$ must be less than $\varepsilon_{2}$, according to IEEE 519 standard [25], the value of $\varepsilon_{1}$ is less than or equal to $5 \%$ and $\varepsilon_{2}$ is less than or equal to $5 \%$. Therefore, to satisfy $\varepsilon_{1}$ and $\varepsilon_{2}$, the objective function $F$ must be less than $5 \%$, then stop and print out the results. Vortex Search algorithm flowchart for designing HAPF is described as in Figure 2.

\section{SIMULATION RESULTS AND DISCUSSION}

To demonstrate the effectiveness of the proposed design method. Let's consider, let us consider a HAPF model as shown in Figure 1. Three-phase source voltage is $380 \mathrm{~V}-50 \mathrm{~Hz}$. THD of the load current is shown in Figure 3.

According to [1], the parameters of the HAPF system with traditional design method are calculated in Table 1. In addition, the controller parameters are randomly selected with the best parameters of $K_{p}=30$ and $K_{i}=0.1$, switching frequency $f=10 \mathrm{kHz}$. 


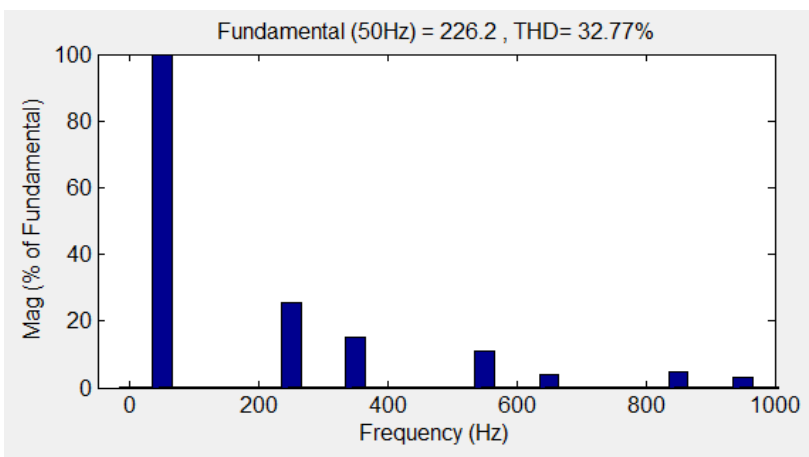

Figure 3. THD of the load current

The parameters in Table 1, we obtain the waveforms of the HAPF system shown in Figure 4. From Figure 4, we see that: THD of the supply current $i_{s}$ decreases from $32.77 \%$ to $2.1 \%$, THD of the source voltage $u_{s}$ is $1.69 \%$, while the reactive power decreased to 140var from $2359 \mathrm{var}$, that is, the capacity compensated by PPFs is 2219var. THD of the supply current $i_{s}$ shown in Figure 5. THD of the source voltage $u_{s}$ is shown in Figure 6.

Table 1. HAPF Parameters by Traditional Design Method

\begin{tabular}{ccccccc}
\hline$C_{F}(\mu \mathrm{F})$ & $C_{l}(\mu \mathrm{F})$ & $L_{l}(\mathrm{mH})$ & $L_{0}(\mathrm{mH})$ & $U_{d c}(\mathrm{~V})$ & $T H D i_{s}(\%)$ & $T H D u_{s}(\%)$ \\
\hline 116.8 & 349.2 & 29.77 & 0.2 & 535 & 2.1 & 1.69 \\
\hline
\end{tabular}
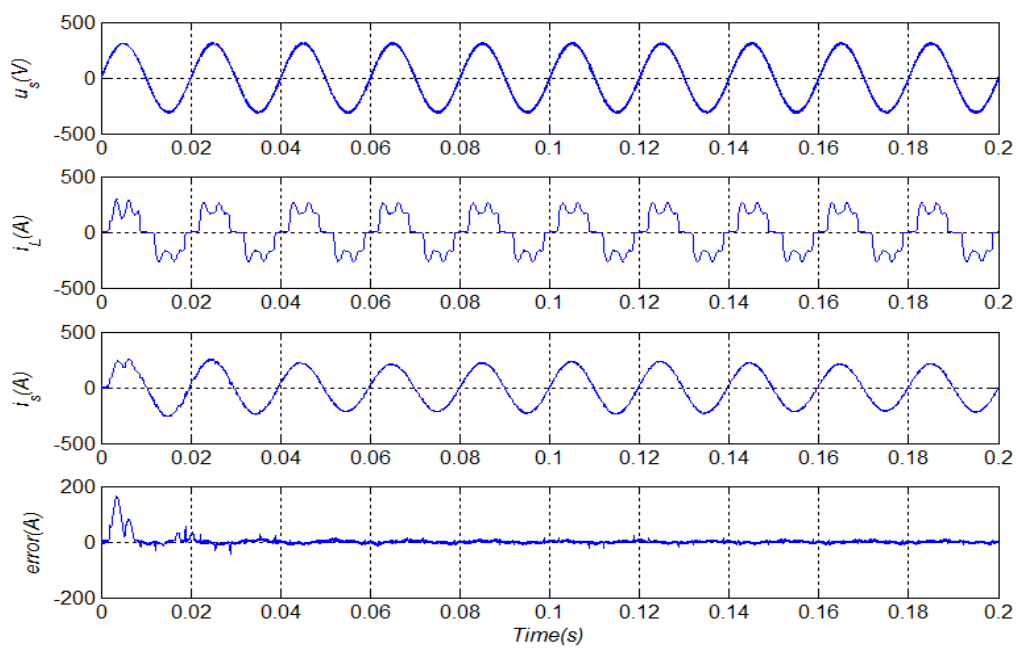

Figure 4. Waveforms of HAPF with traditional design method

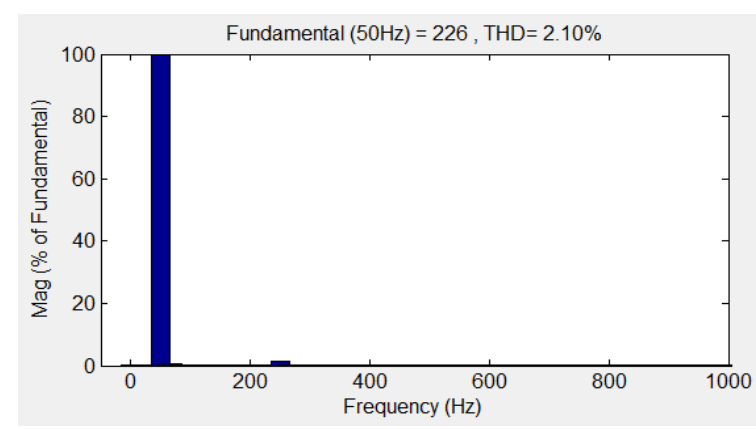

Figure 5. THD of the supply current $i_{s}$

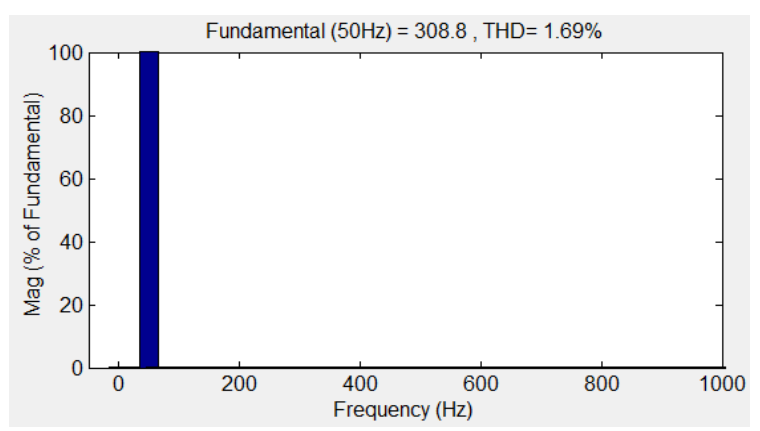

Figure 6. THD of the source voltage $u_{s}$ 
with the proposed design method, the best parameters of HAPF after 10 iterations are shown in Table 2. From the parameters in Table 2, we obtain the waveforms of the HAPF system shown in Figure 7.

Table 2. HAPF Parameters with the Proposed Multi-Objective Optimization Method

\begin{tabular}{ccccccccc}
\hline $\begin{array}{c}C_{F} \\
(\mu \mathrm{F})\end{array}$ & $\begin{array}{c}C_{I} \\
(\mu \mathrm{F})\end{array}$ & $\begin{array}{c}L_{l} \\
(\mathrm{mH})\end{array}$ & $\begin{array}{c}L_{0} \\
(\mathrm{mH})\end{array}$ & $\begin{array}{c}U_{d c} \\
(\mathrm{~V})\end{array}$ & $K_{p}$ & $K_{i}$ & $\begin{array}{c}\text { THD } i_{s} \\
(\%)\end{array}$ & $\begin{array}{c}\text { THD } u_{s} \\
(\%)\end{array}$ \\
\hline 136.67 & 301.22 & 26.6 & 1.01 & 674.13 & 94.95 & 0.65 & 1.14 & 1.28 \\
\hline
\end{tabular}
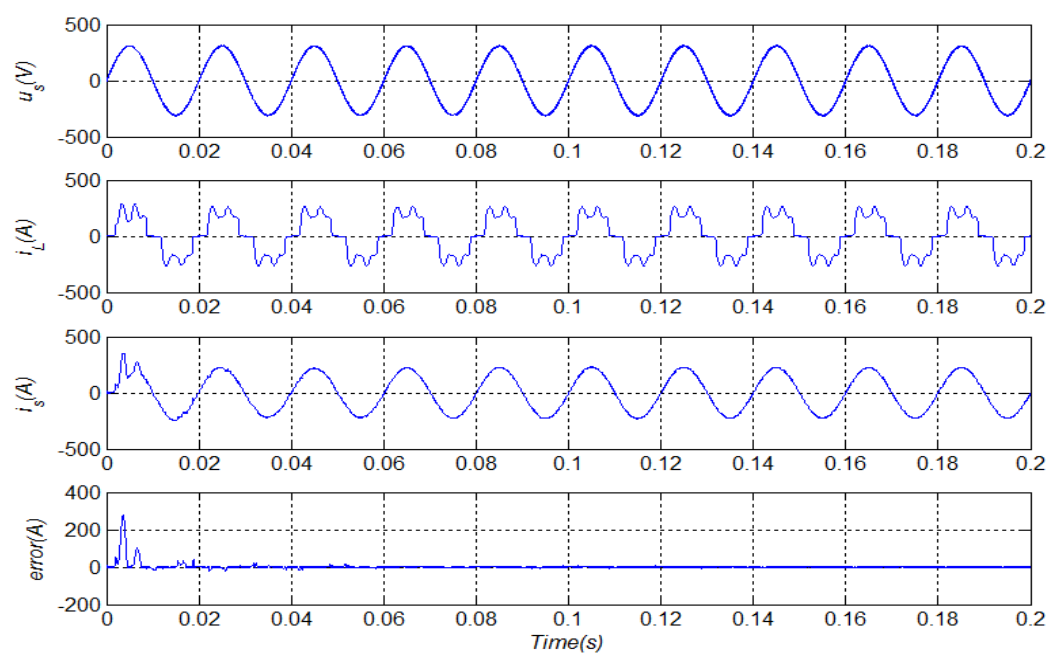

Figure 7. Waveforms of HAPF with the proposed design method

From Figure 7, we can see that: THD of the supply current $i_{s}$ decreases from $32.77 \%$ to $1.14 \%$, while the reactive power decreased to 90var from 2359var, so the compensation power by PPFs is 2269var. THD of the supply current $i_{s}$ in steady-state is shown in Figure 8, THD of the source voltage $u_{s}$ in steady-state is shown in Figure 9.

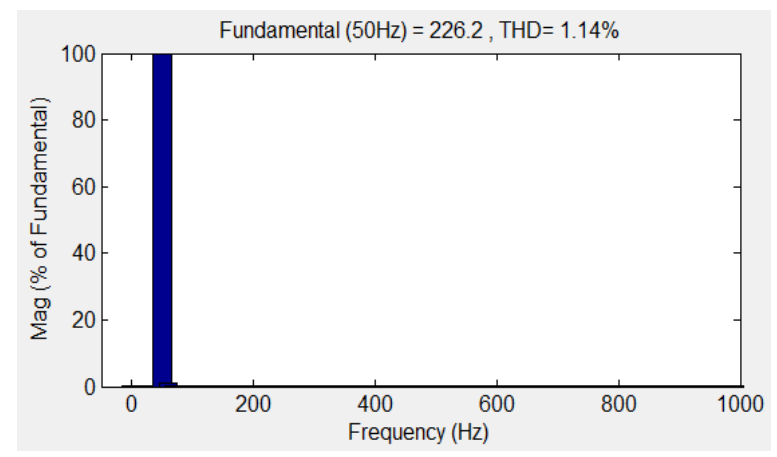

Figure 8. THD of the supply current $i_{s}$

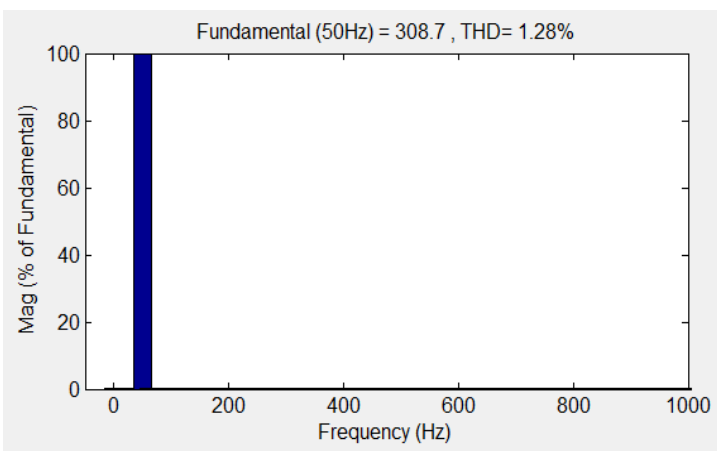

Figure 9. THD of the source voltage $u_{s}$

According to IEEE Std. 519-1992 Recommended Practices and Requirements for harmonic control in electrical power systems [25], then total harmonic distortion of current is less than or equal 5\%. THD of the supply voltage is less than or equal $5 \%$. From the simulation results, we can see that: the proposed design method is more effective than the traditional design method in reducing the total harmonic distortion of the supply current and source voltage and meet IEEE Std. 519-1992. 


\section{CONCLUSION}

The paper has proposed a new multi-objective optimization design algorithm for HAPF based on the Vortex Search algorithm. This method can determine the parameters of both the power circuit part and the control circuit part of the HAPF. Compared to the traditional design method, simulation results have proved that: the achieved results are multi-purpose, such as: minimize the total harmonic distortion of the supply current and source voltage, maximize the reactive power compensation into the grid and satisfying many constraint conditions. This research can be applied to design for all different types of HAPF.

\section{ACKNOWLEDGEMENTS}

This work is supported by the scientific research subject of the Ministry of Industry and Trade of the socialist republic of Viet Nnam No. 081.19.ĐT.BO/HĐKHCN with the title: "Research, design, and manufacture the three-phase active compensation model with $5 \mathrm{~kW}$ capacity for harmonic filtering for nonlinear loads."

\section{REFERENCES}

[1] Chuanping Wu, et al., "Integrated Mathematical Model and Closed Loop Control Characteristic Analysis of Hybrid Active Power Filter," in 2009 International Conference on Sustainable Power Generation and Supply, 2009, pp. 1-7.

[2] Minh Thuyen Chau, "Generalized Current Delay Compensation on Hybrid Active Power Filter," ICIC Express Letters, vol. 11, pp. 1409-1415, 2017.

[3] MinhThuyen Chau, "Stable Self-Adjustment Control Method for Hybrid Active Power Filter," International Journal of Applied Engineering Research, vol. 12, pp. 7162-7168, 2017.

[4] Ibrahim Alhamrouni, et al., "Modeling of Micro-grid with the consideration of total harmonic distortion analysis," Indonesian Journal of Electrical Engineering and Computer Science (IJEECS). vol. 15, pp. 581-592, 2019.

[5] M. Chau, et al., "Online control method with time-delay compensation for hybrid active power filter with Injection Circuit," IET Power Electronics, vol. 5, pp. 1472-1482, 2012.

[6] Minh Thuyen Chau, "Adaptive current control method for Hybrid Active Power Filter," Journal of Electrical Engineering, vol. 67, pp. 343-350, 2016.

[7] An Luo, et al., "Development of Hybrid Active Power Filter Based on the Adaptive Fuzzy Dividing FrequencyControl Method," IEEE Transactions on Power Delivery, vol. 24, pp. 424-432, 2009.

[8] Z. Shuai, A. Luo, C. Tu, D. Liu, "New control method of injection-type hybrid active power filter," IET Power Electronics, vol. 4, pp. 1051-1057, 2011.

[9] Chau Minh Thuyen, "Improved p-q Harmonic Detection Method for Hybrid Active Power Filter," International Journal of Electrical and Computer Engineering (IJECE), vol. 8, pp. 2910-2919, 2018.

[10] H. Akagi, et al., "Generalized Theory of Instantaneous Reactive Power and Its Application," Electrical Engineering of Japan, vol.103, pp. 483-490, 1983.

[11] Y. K. Latha, et al., "Control Strategy for Three Phase Shunt Active Power Filter with Minimum Current Measurements," International Journal of Electrical and Computer Engineering (IJECE), vol. 1, pp. 31-42, 2011.

[12] Pankaj Gakhar and Manoj Gupta, "A novel control strategy for power quality improvement in grid-connected solar photovoltaic system," Indonesian Journal of Electrical Engineering and Computer Science (IJEECS), vol. 15, pp. 1264-1272, 2019.

[13] Chau Van Bao And Chau Minh Thuyen., "DC-Bus Voltage Stabilization of Hybrid Active Power Filter," ICIC Express Letters, vol. 13, no. 1, pp. 27-33, 2019.

[14] An Luo, et al., "Design Considerations for Maintaining DC-Side Voltage of Hybrid Active Power Filter With Injection Circuit," IEEE Transactions on Power Electronics, vol. 24, pp. 75-84, 2009.

[15] C. C. M. Moura, et al., "Determination of the R-L-C parameters of a passive harmonic filter using genetic algorithm," in $10^{\text {th }}$ International Conference on Harmonics and Quality of Power, 2002, pp. 495-500.

[16] Suresh kumar, Ramesh reddy and Archana, "The application of pso to hybrid active power filter design for 3 phase 4-wire system with balanced \& unbalanced loads," International journal of advances in engineering \& technology, vol. 2, pp. 32-42, 2012.

[17] N. S. Hasan, et al., "Harmonic Suppression of Shunt Hybrid Filter using LQR-PSO based," International Journal of Electrical and Computer Engineering (IJECE), vol. 7, pp. 869-876, 2017.

[18] Shengqing Li, et al., "Multi objective optimal design for passive power filters in hybrid power filter system based on multi-island particle swarm optimization," in 7th International Conference on Power Electronics and Motion Control, 2012, pp. 2859-2863.

[19] Suresh kumar and K. Ramesh, "The application PSO hybrid active power filter design for 3 phase 4-wire system with variable load," International Journal of Engineering Inventions, vol. 1, pp. 39-46, 2012.

[20] Nien-Che Yang and Minh-Duy Le, "Multi-objective bat algorithm with time-varying inertia weights for optimal design of passive power filters set," IET Generation, Transmission \& Distribution, vol. 9, pp. 644-654, 2015.

[21] Rrachid Dehini and Slimane Sefiane, "Power quality and cost improvement by passive power filters synthesis using ant colony algorithm," Journal of Theoretical and Applied Information Technology, vol. 1, pp. 70-79, 2012. 
[22] Ahmed Faheem Zobaa, "Optimal multi-objective design of hybrid active power filters considering a distorted environment," IEEE Transactions on Industrial Electronics, vol. 61, pp. 107-114, 2014.

[23] Berat Doğan, Tamer Ölmez, "A new metaheuristic for numerical function optimization: Vortex Search algorithm", Information Sciences, Volume 293, Pages 125-145, 2015.

[24] Doğan B, Ölmez T. "Modified Off-lattice AB Model for Protein Folding Problem Using the Vortex Search Algorithm," International Journal of Machine Learning and Computing. vol. 5, pp. 329-333, 2015.

[25] IEEE Std. 519-1992. IEEE Recommended Practices and Requirements for Harmonic Control in Electrical Power Systems, 1993. 